Drummond, L., Dritschel, B., Astell, A., O'Carroll, R.E. \& Dalgleish, T. (2006). Effects of age, dysphoria and emotion-focusing on autobiographical memory specificity in children. Cognition and Emotion, 20, 488-505.

Effects of Age, Dysphoria and Emotion-Focusing on Autobiographical Memory Specificity in Children

Lyndsey E. Drummond*, Barbara Dritschel and Arlene Astell

University of St. Andrews, Department of Psychology

Ronan E. O’ Carroll

University of Stirling, Department of Psychology

Tim Dalgleish

Emotion Research Group, Medical Research Council, Cognition and Brain Sciences Unit 
This research was supported by a Research Studentship awarded to Lyndsey Drummond by the University of St. Andrews (supervised by Barbara Dritschel). Tim Dalgleish is supported by the U.K. Medical Research Council.

*Correspondence concerning this article should be addressed to Lyndsey Drummond, School of Psychology, University of St. Andrews, St. Mary’s Place, Scotland KY16 9JU. E-mail: led@st-andrews.ac.uk. 
Overgeneral Autobiographical Memory (ABM) is strongly associated with depression in adults and appears to reflect a stable cognitive bias. However it is not known whether this bias exists in children or what factors contribute to its development. We examined the roles of age, dysphoria and a new variable, Emotion-Focusing, on the production of specific ABM in children, using the standard ABM cuing task. Results show that older children were more specific than younger children, irrespective of cue valence. Dysphoria was linked to overgeneral retrieval of positive memories in children. A 3-way interaction between age, valence and dysphoria was also found, such that older dysphoric children demonstrate a difficulty in retrieving specific negative memories. In addition, Emotion-Focusing was associated with specific ABM recall, especially to negative cues. Results are discussed with reference to the development of depressogenic biases. 


\section{Effects of Age, Dysphoria and Emotion-Focusing on Autobiographical Memory Specificity in Children}

Depression in adults is reliably associated with an overgeneral autobiographical memory (ABM) retrieval style (van Vreeswijk \& de Wilde, 2004). Evidence suggests that overgeneral retrieval exists as part of a stable, dysfunctional cognitive style and is somewhat independent of current mood (e.g. Brittlebank, Scott, Williams \& Ferrier, 1993; Mackinger, Pachinger \& Leibertseder, 2000). This has led a number of theorists to assert that the roots of this bias may lie in childhood. Williams (1996) proposed that an overgeneral memory style develops from early childhood as a means of coping with negative affect. Individuals, for example, who have experienced trauma during childhood, have greater difficulty in retrieving specific memories in adulthood, even for events unrelated to the trauma (e.g. Kuyken \& Brewin, 1995). Adolescents exposed to family violence during childhood, and reporting co-morbid depression, also exhibit an overgeneral memory bias as evidenced in interview data concerning family conflict (Orbach, Lamb, Sternberg, Williams \& Dawud-Noursi, 2001). Though links between development of an overgeneral memory style and depression have been made in theory, to date there are no studies examining overgeneral memory bias in children. Importantly, if overgeneral recall does reflect a stable bias, it could be a useful marker of cognitive vulnerability to depression in children.

We have some insight into the development of the ABM retrieval bias from investigations involving adolescents (de Decker, Hermans, Raes \& Eelen, 2003; Park, Goodyer \& Teasdale, 2002; Swales, Williams \& Wood, 2001). In these studies, as expected from the adult literature, clinically depressed adolescents are more over-general in their responses to cue words than non-depressed controls. However, in the Swales et al. study there was also a positive 
correlation within the clinical population, with the more depressed and hopeless adolescents demonstrating greater specificity to negative cues. This is in the opposite direction to that which would be expected from previous findings with depressed adults (van Vreeswijk \& de Wilde, 2004). Post-hoc analyses attributed this finding to a repeated memory phenomenon, whereby a sub-set of parasuicidal adolescents recalled the same negative traumatic event to more than one cue word. The researcher was the therapist in this study, which may also be pertinent to the result. Nevertheless, repeated specific recall can be addressed with clear participant instruction to prevent or judge repetition.

Studies examining ABM retrieval in children have tended to concentrate on the onset of autobiographical memory recall in pre-school to early school age children and have used different methodologies from those used in the adult literature. This developmental literature reveals that specific questions can yield specific ABM responses even in very young children. However, overall, young children's preferred mode of memory reporting is general (Nelson, 1993). Greater detail, structure and reference to temporal markers are then observed in ABMs as a function of increasing age (Howe \& Courage, 1997). These findings are discussed in terms of language acquisition (Nelson, 1993) and self-concept formation (Howe \& Courage, 1997) rather than in terms of specificity or generality per se. However, improvement in knowledge base, strategy use, language skill, self-concept and storage capacity (for a full review see Howe \& Courage, 1997) are likely to facilitate ABM response specificity. By age 7 the quality of children’s ABM recall is considered comparable with adult functioning (Gathercole, 1998). The present study therefore sought to test an early to middle-school age child population on the standard ABM cuing task in order to bridge the gap in the ABM and depression literature and to look for evidence of the development of or onset of an overgeneral retrieval style prior to its 
establishment in adolescence. The use of the standard ABM cuing methodology then makes direct comparison between child and adult retrieval styles possible.

We considered that age and dysphoria might also interact to affect ABM production. In a 5-year longitudinal study of 8-13 year olds, Nolen-Hoesksema, Girgus and Seligman (1992) found that as children age, cognitive style as measured by the Children's Attributional Style Questionnaire (Kaslow, 1978), became a significant predictor of depression. In contrast, only negative life events predicted depression in the younger group. Authors suggest the reason for this apparent increasing role of cognitive style in depression with age may be due to cognitive capacities increasing with age, or because cognitive styles become more stable across later childhood. Similar data exist showing an increasing relationship between depression and mood congruent memory bias with age (Neshat-Doost, Taghavi, Moradi, Yule \& Dalgleish, 1998). If age, mood and cognition do interact in this way, the association between dysphoria and overgeneral retrieval may manifest itself differently in younger as opposed to older children.

A further factor that might interact with age and dysphoria to influence ABM retrieval is cue valence. In the adult literature overgeneral recall can occur more in response to positive as opposed to negative cue words (e.g. Williams \& Broadbent, 1986) or it can show the reverse effect (e.g. Mackinger, 2000). Inconsistent valence effects in depressed groups may be attributable to variance in the relative casual contributions of affect regulation (Williams 1996), temporary memory system disruption caused by schema activation (Dalgleish, Yiend, Tchanturia, Serpell, Hems, De Silva \& Treasure, 2003) or mood congruency (see Williams, Watts, MacLeod \& Matthews, 1997). All are likely to play a role. It is also possible that age further influences this valence effect. Drummond, Dritschel, Astell and O’Carroll (in review) tested children aged 6-11 years and a young adult comparison group on perception of facial 
affect. Ability to recognize facial emotion increased with increasing chronological age in this study. The results showed that there was no significant difference between adults and children on recognition of happy or neutral faces. However there was a significant difference between children and adults on rating of sad faces. Children were significantly poorer than adults on identification of negative facial affect. Children made many 'sad as neutral' type errors, suggesting there might be an important developmental effect of processing of negatively valenced material in children. An aim of the present study was to further investigate this issue.

Finally, this study is the first to introduce the idea of 'Emotion Focusing' (EF) as a possible additional variable influencing the overgeneral memory effect. Motivation for studying this variable comes from Williams (1996) who argued that overgeneral memory may in part, be attributable to preferential encoding of situations according to their emotional content. Williams (2000) re-states that trauma in early childhood may result in a cognitive style in which individuals habitually focus on or are hypersensitive to the affective features of events. The extent to which individuals focus on (encode) the emotional features of their environment is an important factor that has hitherto been neglected in both child and adult investigations of ABM retrieval. EF was therefore used in this study to denote individuals' predisposition to focus on the emotional features of their environment. For example, given a typical scenario of a university library during an exam period, one individual may encode the fact that several students at the desks look terrified and tearful, while another individual, viewing the same scene, may notice instead that there is a pile of precariously stacked books against a chair leg. The first individual will be effectively cued to remember the library event by the word 'fear' while the second individual would be better cued by the phrase 'safety-hazard' and would probably not remember the event based on an emotional descriptor. 
Regarding ABM predictions concerning EF, Williams (1996) suggested that people who have a greater tendency to encode the emotional content of situations would be more likely to demonstrate overgeneral recall than those who do not. This argument is based on the assumption that focusing on emotion promotes schematic level encoding. Overgeneral autobiographical recall would then result in individuals who were sensitised to emotion during childhood and therefore may encode emotion preferentially, for example in the case of early childhood trauma. This hypothesized link between EF, overgeneral recall and vulnerability to depression has not as yet been investigated.

Other theoretical accounts of cognition-emotion relations may however, make different predictions. For example, cognitive theories of depressed mood and/or autobiographical memory (Bower, 1981; Beck et al., 1979; Conway\& Pleydell-Pearce, 2000; Foa \& Kozak, 1986; Power \& Dalgleish, 1997; Teasdale \& Barnard, 1993) would suggest that, to the extent that a specific autobiographical event is encoded in emotional terms, the presence of a congruent emotional cue word (such as in the AMT) would be more likely to elicit retrieval of that event from memory. The rationale here is that retrieval of ABMs is facilitated by the presence of any information that maps onto the underlying representational content. Consequently, because high EF individuals would habitually lay down ABM representations with a greater proportion of emotional content, the presentation of emotional cues should be more likely to activate those representations leading to facilitated generation of specific memories. An alternative hypothesis for the effect of EF on ABM retrieval is therefore that individuals' predisposition to focus on the emotional features of their environment may result in more specific ABM recall to emotional cue words. People who are depressed may then preferentially encode negative affective aspects of events such that 
depressed, high emotion-focusers would be highly specific to negative cues. It is currently not known whether EF is associated with overgeneral or specific valenced recall.

In sum, although overgeneral ABM is generally discussed as a retrieval phenomenon, it is also useful to consider it as a possible encoding phenomenon. It is not clear that children or adults encode all information in a given context and then later suppress specific aspects at retrieval. Instead, some individuals may avoid or preferentially encode specific features (or emotional features) of events such that it effects specific representations in memory. This would mean that both encoding and storage (not just retrieval) are important to overgeneral recall. Whether considered in isolation, or as interacting with other factors such as age, dysphoria or valence, EF may significantly influence the nature of ABM recall and thereby, the development of depressive associated biases. The overall aim of this study then was to extend the adult ABM literature to a child population. We looked at how age, dysphoria, cue valence and EF may influence autobiographical memory specificity in children. To our knowledge, no one study has looked at the interaction of age, dysphoria and valence as factors known to influence ABM, nor has any study considered EF as a contributing factor. The specific hypotheses were therefore as follows:

Hypothesis 1: Age, valence and dysphoria will interact to influence ABM specificity in children. Younger children will be less specific than older children. Age and valence will interact such that young children will demonstrate reduced specificity especially to negative cue words. Dysphoria in children will be associated with reduced specificity and the effects of dysphoria will be valence specific. The effects of dysphoria may increase with age. These effects will remain after controlling for any effects of general scholastic ability. 
Hypothesis 2: EF will explain a significant proportion of the variance in ABM specificity. EF will be associated with either greater specific or greater overgeneral ABM recall. EF will also interact with age, valence or dysphoria, factors already known to influence ABM recall. The effects of EF will occur independent of scholastic ability or age.

\section{METHOD}

Participants

Seventy children (35 girls) took part in this study. Thirty-five of the participants were aged 7-8 years (mean $7.86, \mathrm{SD}=.40$ ). The remaining thirty-five participants were aged 10-11 years (mean $=10.6, \mathrm{SD}=.72)$. The gender ratio across age groups was similar with 17 females in the younger group and 18 females in the older group. As previously discussed, children aged 7-8 years were chosen as the youngest age group for which ABM retrieval is believed to be commensurate with adult functioning. Children aged 10-11 were then chosen to provide a discrete age comparison group and to form a good bridge with previous adolescence literature (e.g. Park et al., 2002). These two age groups ensured the best chance of detecting deviance in normal child ABM development if any exists. There were 14 dysphoric participants (8 from the younger group and 6 from the older group). The remaining participants (27 and 29 respectively) were categorized as non-dysphoric. 'Dysphoric' in this study means reporting a depressive mood (as indicated by a score of 7 or above on the Child Depression Inventory; Kovacs, 1992, see below).

All participants were volunteers recruited from a London, UK Junior School. The school was initially contacted to sanction the research proposal. Research approval was then obtained from the relevant ethical committees. Permission to conduct research was sought from individual classroom teachers, followed by a letter of consent to parents and finally written consent from 
the children themselves. The sample was ethnically mixed. No individuals with Special Educational Needs were included in this study. No child with any known history of trauma or emotional disturbance, as established from school records and teacher reports was included. This is because a history of trauma is known to influence ABM recall (e.g. de Decker et al., 2003) and there would have been insufficient children with a history of trauma to examine any such influences systematically.

Materials and Measures

ABM. The standard ABM cuing test was used (Williams \& Broadbent, 1986). The task comprised 10 emotional words, 5 positively valenced (happy, surprised, safe, successful, interested) and 5 negatively valenced (sad, lonely, hurt, careless, angry). Words were presented to participants on $120 \mathrm{~mm}$ x $100 \mathrm{~mm}$ laminated cards and participants were asked to retrieve a specific memory for each cue word. All participants completed a practice trial involving 3 neutral words with feedback, to ensure that all participants understood and were able to complete the requirements of the task. Every child retrieved at least one specific memory during the practice trial. Participants were informed that that they would be prompted to provide a specific memory if an overgeneral response was given (i.e. a memory that was not of a particular event on a given day). In addition, clear instruction was given requesting that the child not give the same memory to more than one cue word to avoid the phenomenon of 'repeated memories' discussed earlier. The presentation of cue words was mixed across valence. Participants were given 60 seconds to retrieve a memory. Failure to retrieve any memory was coded as a memory omission. Failure to retrieve a specific memory resulted in the allocation of an overgeneral 
response categorisation for that cue word. All participant responses were recorded on audiotape and later coded for specificity.

Two independent raters coded the ABM responses as specific, overgeneral (extended or categoric) or no memory. An example of scoring of an overgeneral memory response to the cue word 'Lonely' was, “When I was in Year Two I used to sit on the lonely bench every play time”. This was coded as overgeneral because it is a categoric memory. A specific memory response to the same cue was, “On Friday- When I was at home. I had no-one to play with 'cause my brothers were out at a friends and I wasn't allowed to go”. This memory was coded as a specific response because the memory is of a particular place and time (of less than one day). Over 85\% inter-rater reliability was achieved on coding of these responses. This reliability estimate is comparable to reliability rates found in the adult literature (e.g. Williams \& Broadbent, 1986).

Validity of the AMT for use in children was established in several ways. No child failed to retrieve a specific ABM to at least one practice cue word and some of the experimental cue words. Although some of the children in the youngest age group were unsure of the meaning of the words 'successful' or 'careless', in these instances $(\mathrm{N}=4)$ the experimenter explained the meaning of the word by invariably saying 'good at something' or 'clumsy'. This was sufficient for all children to acknowledge their understanding of the word and quickly retrieve a valid memory (specific/overgeneral). There were only 7 memory omissions in total (3 from older children). Content validity was also examined by having two raters judge whether the content of the reported memory was appropriate to the cue word used to elicit the memory. All children recalled appropriate content memories. High content validity was confirmed by over 95\% interrater agreement on memory content appropriateness on a random $50 \%$ of the sample. 
Emotion Focusing. A measure of EF was constructed using combined results from two tasks: Card Sorting and Image Description. The Card Sorting Task consisted of a set of 12 cards (90 mm x $60 \mathrm{~mm}$ ) each portraying an adult face displaying one of three different emotional facial expressions (happy, neutral or sad). The faces were arranged in a $6 x 2$ formation and consisted of an equal gender mix, mixed age and appearance e.g. hair length, colour. The faces were not of mixed race due to limited availability. All photographs were drawn from a sample set of faces, which had previously been used and received over 75\% inter-rater agreement concerning the emotion being portrayed (Le Gal \& Bruce, 1999). Firstly, the experimenter verified that each child understood the nature of grouping and physically demonstrated that the cards could be sorted into two groups, using gender as the most obvious sorting-category. Once established, the cards were then replaced and the children were required to sort the cards according to their own sorting category, verbalising the decision as soon as one was made. A score of 1 was given when the child sorted the faces according to affect (emotion). A score of zero was given for any other (non-emotional) sorting category. The sorting categories suggested by children were emotion (happy and sad faces), hair colour, hair length, age, face-shape and subjective attractiveness.

The second EF task was the Image Description Task. This involved a picture-cuing methodology. A series of 11 photographic images of socio-emotional behavioural settings e.g. family laughing around a dinner table, child being admonished by parent figure, were drawn from a developmental psychology stimulus pool. The 11 images were presented to the participants as $200 \mathrm{~mm}$ x $150 \mathrm{~mm}$ laminates, and depicted for example, 'Parents standing over a baby, smiling'. All but one of the cards involved a child protagonist. Protagonists were of mixed age and race. Participants were simply asked to 'Tell me what you see' in response to being shown each pictorial card. The participant responses were recorded and later coded for emotional 
description. Subjects were given a score of 1 if an emotional adjective or adverb was used in the description of the card, for example 'happy, excited, loving'. A score of zero was given for each card where no emotional description was given, for example ‘a baby, a mum and a dad.' Two raters examined participant responses in terms of these categories. When emotion was implied but an emotional adjective was not directly used e.g. 'running hard' or 'dad is making up with son for missing him play football', it was decided that a strict criterion would be used and only an emotional descriptive would result in an emotional classification. That is, inference of emotion was not sufficient to classify as an emotional description. Using this criterion, 100\% inter-rater category agreement was achieved.

From these two tasks a combined measure of EF was constructed whereby the sample was divided into high, medium or low emotion-focusers according to the following criteria. The criteria for low EF was that the participant neither sorted the cards by emotion nor used emotion descriptions in over $75 \%$ of the images in the image description task. The criteria for medium EF was that the participant either sorted the cards by emotion or described over $75 \%$ of the images in terms of emotion. The criteria for high EF required the participant to both sort the cards by emotion and describe over $75 \%$ of the images in terms of emotion. This classification was considered statistically reasonable in the absence of available theoretical or empirical constraints.

Child Depression Inventory. The shortened version of the Child Depression Inventory (CDI-S, Kovacs, 1992) was completed. This measure consists of 10 mood related items with three possible responses per item. Participants are directed to indicate which of each of the 3 statements best applies to them over the last 2 weeks. The statements are read out loud to the participants and participants are required to tick the most appropriate statement to them.

According to CDI guidelines (Kovacs, 1992) participants with a CDI score of 7 or above are 
classed as dysphoric and those with scores below 7 are classed as non-dysphoric. The word 'dysphoric' is used in this study rather than 'depressed' simply to stress that the current authors felt the measure best reflects a depressive mood or emotional state characterised by depression, which can be transient, rather than a depression disorder, which should be formally diagnosed.

General Ability. A measure of scholastic ability was constructed for each child using a combination of teacher report and age appropriate National Curriculum Attainment levels. This is a method of ability grouping commonly used in British primary school education. From these two sources, children were divided into high, medium and low ability groups. Where discrepancy existed between groupings based on optional SAT levels, e.g. high attainment in Maths but medium attainment in English, the teacher's summative assessment on overall ability was used as the final criterion. Though Standard IQ is more commonplace and therefore an easier measure to interpret across studies, the measure of scholastic ability was favoured to respect testing time constraints, to avoid unnecessary stress caused to individuals by IQ testing in a young and partly dysphoric sample, and to utilize teacher reports of child potential for an arguably stronger index of actual ability.

Procedure

Participants were tested individually and face to face, in a quiet testing environment within the school. Participants were first asked to complete the ABM cuing task, followed by the two EF tasks and ending with completion of the CDI. Task order was fixed to avoid possible contamination effects of the CDI or EF on the ABM measure. Once the participants had completed all 4 experimental tasks they were thanked for their participation and fully debriefed. 
After the testing session an indicator of general scholastic ability was obtained from school records and consultation with the appropriate class teacher.

\section{RESULTS}

The numbers of specific ABMs by age, dysphoria and cue valence are shown in Table 1. In keeping with predictions, the data in Table 1 indicate that young non-dysphoric children are less specific to negative cues than to positive cues. Furthermore young dysphoric children appear to recall more specific negative memories and fewer specific positive memories compared to their non-dysphoric age equivalents. The data also indicate that dysphoric children in the older group are less specific than older non-dysphoric children to both positive and negative cues, suggesting evidence of the overgeneral memory bias.

\section{Table 1 about here.}

A three way ANOVA with Age (7-8 yrs and 10-11 yrs), Dysphoria (high and low) and Valence (positive and negative) was conducted to examine this assumption. General Ability (high, medium and low) was initially included as a covariate but was not significant, $\mathrm{F}_{(1,65)}=$ $1.56, \mathrm{P}=.22$. Reported analyses are therefore without this covariate. The ANOVA revealed a significant main effect of Age, $F_{(1,66)}=38.80, p<.001$, such that older children were more specific than younger children. Results also revealed a main effect of Dysphoria, $F_{(1,66)}=7.38$, $\mathrm{p}=.01$, such that overall dysphoric children were less specific than non-dysphoric children. There was also a Valence trend, $\mathrm{F}_{(1,66)}=3.77, \mathrm{p}=0.056$, in that children tended to recall fewer specific 
negative memories than positive. However, these main effects were qualified by a 3-way interaction between Age x Valence x Dysphoria on ABM recall $\left(F_{(1,66)}=4.4, p=.04\right)$.

In order to deconstruct this 3-way interaction, three 2-way ANOVAs were conducted. The first examined the relationship between Age and Dysphoria on recall to positive cues, the second looked at the relationship between Age and Dysphoria on recall to negative cues. A third ANOVA was conducted to directly examine the relationship between Age and Valence on ABM recall. In

the first 2x2 ANOVA (Age x Dysphoria) on specific positive recall there remained a main effect of Age, $\mathrm{F}_{(1,65)}=18.25, \mathrm{p}<.001$, such that younger children recalled fewer specific positive memories than older children and a main effect of Dysphoria, $F_{(1,65)}=20.75, p<.001$, such that dysphoric children recalled fewer specific memories to positive cues than non-dysphoric. There was no significant interaction between Age and Dysphoria on positive recall $\left(\mathrm{F}_{(1,65)}=.26, \mathrm{p}=\right.$ $.63)$.

In the second 2x2 ANOVA (Age x Dysphoria) on specific negative recall there remained a main effect of Age, $F_{(1,65)}=27.31, \mathrm{p}<.001$, such that young children recalled fewer specific negative memories than older children. There was no main effect of Dysphoria, $\mathrm{F}_{(1,65)}=.04$, $\mathrm{p}=.85$. However, there was a significant interaction between Age and Dysphoria, $\mathrm{F}_{(1,65)}=7.79$, $\mathrm{p}=.007$, such that non-dysphoric children's recall of negative memories significantly improved across age ( $\mathrm{t}(54)=10.21, \mathrm{p}<.001)$, while dysphoric children's negative specific recall, though higher than non-dysphorics in the younger group, stayed static across age $(\mathrm{t}(12)=.97, \mathrm{p}=.35)$. Moreover, by age 10-11 dysphoric children recalled significantly fewer specific memories to 
negative cues than their non-dysphoric age equivalents $(\mathrm{t}(33)=2.4, \mathrm{p}=.02)$, in keeping with an overgeneral memory bias.

In order to directly examine the relationship between Age and Valence a 2x2 ANOVA (age x valence) was conducted. Results show a main effect of Age, $F_{(1,68)}=74.92, p<.001$, a main effect of Valence, $F_{(1,68)}=20.17, \mathrm{p}<.001$, and a significant interaction between Age and Valence, $\mathrm{F}_{(1,68)}=13.22, \mathrm{p}<.001$ such that older children were more specific than younger children to both positive $(\mathrm{t}(68)=4.77, \mathrm{p}<.001)$ and negative cues $(\mathrm{t}(68)=8.32, \mathrm{p}<.001)$. However, in the younger group children recalled significantly fewer memories to negative cues compared to positive $(\mathrm{t}(34)=5.45, \mathrm{p}<.001)$. This valence difference was not significant in the older group $(\mathrm{t}(34)=.64, \mathrm{p}=.53)$.

In summary, age affected positive and negative recall such that older children were more specific to both. Dysphoria exerted a main effect on positive recall such that dysphoric children retrieved fewer specific positive memories than non-dysphoric children. The 3-way interaction above was attributable to age and dysphoria interacting to influence the recall of negative memories. Young non-dysphoric children showed low specificity to negative cues. Older nondysphoric children showed relatively high negative specificity. In contrast, young dysphoric children showed higher specificity to negative cues than their same age counterparts and yet specificity to negative cues in the older dysphoric group was comparatively low.

The following analyses addressed the second hypothesis, which was that EF would contribute to ABM specificity and may interact with other factors to influence specificity. This is the first study to examine EF. It was therefore important firstly to establish that EF was not simply a correlate of another variable such as age or scholastic ability, which would reduce the usefulness of EF as a measure. There was however no correlation between EF and Age (rho 
$=.13, \mathrm{n}=70, \mathrm{p}=.27$ ); General Scholastic Ability (rho =.08, $\mathrm{n}=70, \mathrm{p}=.51$ ) or Dysphoria (rho $=.12$, $\mathrm{n}=70, \mathrm{p}=.3$ ) in this sample.

Subsequently, an enter-stepwise regression model was conducted on total specific ABM such that Age and Dysphoria were entered in a first step and EF was added in a second step, to investigate whether EF accounted for unique variance in ABM specificity, over and above any effects of age and dysphoria. Age and Dysphoria accounted for significant variance in ABM specificity $\left(\mathrm{F}_{(2,69)}=45.69, \mathrm{p}<.001\right.$, Adjusted $\left.\mathrm{R}^{2}=.56\right)$. The inclusion of EF resulted in a significant additional $12 \%$ of variance being explained $\left(\mathrm{R}^{2}\right.$ change $\left.=.12, \mathrm{~F}=25.05, \mathrm{p}>.001\right)$. The final model included Age as the best independent predictor of specificity $(r=.72, n=70$, beta .66 , $\mathrm{p}<.001)$, followed by EF $(\mathrm{r}=.47, \mathrm{n}=70$, beta .35, $\mathrm{p}<.001)$ and then Dysphoria $(\mathrm{r}=.07, \mathrm{n}=70$, beta $.27, \mathrm{p}<.001)$. All three variables contributed to a significant final model accounting for $68 \%$ of the variance in specificity $\left(\mathrm{F}_{(3,69)}=49.75, \mathrm{p}<.001\right.$, Adjusted $\left.\mathrm{R}^{2}=.68\right)$. There was no collinearity between variables $(\mathrm{VIF}<2)$.

Given that EF was shown to be an important predictor of ABM specificity, we repeated the analyses used to test Hypothesis 1, this time including EF as an additional factor. Two 3x2x2 ANOVAs with EF (high, medium and low), Age (7-8 yrs and 10-11 yrs) and Dysphoria (dysphoric and non-dysphoric) were therefore conducted, first for positive and then for negative valence, to test the hypothesis that EF would interact with other factors known to influence ABM performance.

\section{Table 2 about here.}

Once again results of the analysis of positive recall showed the main effects of Age, $\mathrm{F}$ $(1,58)=18.68, \mathrm{p}<.001$ and Dysphoria, $\mathrm{F}_{(1,58)}=28.95, \mathrm{p}<.001$. As can be seen in Table 2 , there was 
no main effect of EF on positive recall, $\mathrm{F}_{(2,58)}=1.79, \mathrm{p}=.18$. However, a significant interaction was found between EF and age on positive recall, $\mathrm{F}_{(2,58)}=4.29, \mathrm{p}=.018$, such that older children were more specific than younger children in the low and medium EF categories (t $(17)=3.04$, $\mathrm{p}=.007$ and $\mathrm{t}(28)=4.13, \mathrm{p}<.001$, respectively). However, in the high EF group there was no significant difference between younger and older children's specific positive recall (t (19) =-.91, $\mathrm{p}=.38$ ). Young children are just as likely to produce a specific positive memory as older children in this group. There was no significant 3-way interaction between EF, Age and Dysphoria (F (2, $\left.{ }_{58}=0.25, \mathrm{p}=.78\right)$ and no interaction between EF and Dysphoria $\left(\mathrm{F}_{(2,58)}=1.02, \mathrm{p}=.37\right)$.

When EF was included in an analysis of specific negative recall, there remained a main effect of Age $\left(F_{(1,58)}=35.18, p<.001\right)$ with no main effect of Dysphoria $\left(F_{(1,58)}=1.41, p=.24\right)$. However EF exerted a main effect on negative recall, $\mathrm{F}_{(2,58)}=17.02, \mathrm{p}<.001$, such that high emotion focusers were more specific to negative cues compared to low $(\mathrm{t}(38)=6.12, \mathrm{p}<.001)$ or medium ( $(48)=3.70, \mathrm{p}<.001)$ emotion focusers. EF did not interact with age to affect negative recall $\left(\mathrm{F}_{(2,58)}=1.74, \mathrm{p}=.18\right)$ nor was there any 3-way interaction between EF, Dysphoria and Age $\left(\mathrm{F}_{(2,58)}=1.22, \mathrm{p}=.30\right)$. Of interest to us was whether EF and Dysphoria would interact significantly to influence negative specificity however, no such interaction was found $\left(\mathrm{F}_{(2,58)}=\right.$ 1.68, $\mathrm{p}=.20)$.

\section{DISCUSSION}

The results of this study show that age, dysphoria and cue valence interact to influence ABM specificity in children. Older children are more specific than younger children especially to negative cues. Dysphoric children are overgeneral to positive cues and there is an interaction between age, cue valence and dysphoria such that older dysphoric children are also overgeneral 
to negative cues. Regarding EF, high emotion-focusers produce specific ABMs and EF significantly predicts unique variance in ABM specificity. Each of the main findings is discussed in turn and possible mechanistic implications are considered.

First, as predicted, older children were able to produce a specific memory more readily than younger children. This suggests that between 7 and 11 years of age, a child's autobiographical recall develops from a general to a more specific memory style. This is consistent with the finding in the developmental literature that memorial ability develops with age (Nelson, 1993; Howe \& Courage, 1997). As predicted, this effect of age on specificity was further influenced by cue valence. Younger children produced fewer specific memories in response to negative cues than to positive. This difference was not apparent with the older children, who presented approximately equal numbers of positive and negative specific memories. This age by valence effect has not been previously demonstrated and it suggests that it is particularly children's specific negative recall, which improves with age. It is clear that the development of cognitive processing of valenced stimuli in children is an interesting and previously neglected area of research, which could hold clues to the origins of information processing biases associated with depression. In addition, if autobiographical memory recall in a normal population develops from a general to a specific retrieval style with age, the question remains whether in a depressed population memory fails to develop into a specific style or whether depressed individuals regress back to an overgeneral style.

Our results are the first to provide direct evidence that dysphoria is linked to overgeneral recall in children. Dysphoric children retrieved significantly fewer specific positive ABMs than non-dysphoric children, irrespective of age. This is consistent with the well-documented adult ABM literature and with adolescent findings, in general. Moreover, age and dysphoria interacted 
to affect specific negative retrieval such that there was an effect of dysphoria on negative recall in the older group. Dysphoric children in the older group retrieved significantly fewer specific negative memories compared to their non-dysphoric age equivalents. What is more, when we examined the pattern of negative ABM retrieval across groups more closely, we found that the younger dysphoric children retrieved more specific negative memories than their non-dysphoric age-group counterparts. However, while non-dysphoric negative recall significantly improved across age group, the same was not true for dysphoric children. By age 10-11, therefore, dysphoric children were retrieving significantly fewer specific negative memories than nondysphoric older children. Thus, though dysphoric children demonstrate reduced specificity for both negative and positive cues, the effects of dysphoria on negative recall are only really apparent in the older group.

It may be that dysphoria affects positive and negative recall through different underlying mechanisms or processes. For instance, dysphoria may be affecting positive recall via mood congruency, which is a relatively autonomous mechanism based on spreading activation and therefore less dependent on age or cognitive ability than other possible mechanisms. In contrast, the impact of dysphoria on negative recall may be more schema dependent, and therefore its effect on recall is not observed until later in childhood when schemas are more firmly in place. In keeping with this schema explanation, repeated experience of dysphoric mood may allow negative biases to become more engrained and better established in older dysphoric children. In younger dysphoric children however, negative schemas may be less well defined and therefore exert less influence on information processing. This interpretation, though speculative, is supported by results from Nolen-Hoeksema et al. (1992), which showed that effects of cognition on depression increased with increasing age and also with results from Neshat-Doost et al (1998) 
who found evidence suggestive of an age-related increase in the elaboration of negative selfreferent schematic representations in depressed youth. Predictions from kindling and sensitization theory would also lend credence to this type of interpretation (see for example Segal, Williams, Teasdale \& Gemar, 1996).

Alternatively, it may be that a young child's negative ABM response style is already so highly overgeneral that dysphoria can exert little additional effect. It is therefore not until later in childhood, when children have typically developed a more specific retrieval style, that the overgeneral effect of dysphoria on negative recall would become apparent. Interestingly, this relative difficulty in retrieving specific negative memories in older dysphoric children is not in keeping with the finding that depressed adolescents demonstrate highly specific recall to negative cues (e.g. Park et al., 2002). Further research is needed to clarify the differences in valence specific ABM styles in children, adults and adolescents.

Our study also introduced the idea of Emotion-Focusing as a potential vulnerability marker for depression. EF significantly contributed to predicting variance in ABM specificity, even above the predictive value of age and dysphoria. Our results indicate that high emotionfocusers demonstrate specific memory recall to emotional cue words. Thus, an emotion-focuser who preferentially focuses on emotion in the environment may form memory representations with relatively high emotional content. When these individuals are then asked to recall events in response to emotional cue words, specific retrieval is then relatively easy for these individuals. EF could be important especially if attempts are made to assist people in recalling specific ABM information something that is a core component of some cognitive based clinical treatments. Emotion encoding does not appear to correspond with overgeneral retrieval as expected by Williams (1996). However it is proposed that with the addition of an environmental stressor, EF 
may emerge as a vulnerability factor for depression, more in keeping with the trauma-induced EF hypothesis proposed by Williams. EF is clearly a meaningful new measure for use with children and adults and warrants further investigation.

It is important to note that emotion focusing was not correlated with any measured independent variable in this study. Though there was an interaction between age and EF on positive recall, there was no co-linearity with this variable. EF was not a product of age nor was there any correlation between scholastic ability or dysphoria, and EF. Therefore the variability accounted for by EF in this study is clearly not attributable in any straightforward way to the effects of dysphoria, age or ability. Further longitudinal studies are required to look more closely at the relationship between negative life events, EF and ABM retrieval over time. It may be, for example, that EF interacts with ruminative style to affect depressive mood.

Furthermore, in order to develop the measure of EF, a greater range of stimuli could be added to the image description element or a more sophisticated coding system could be introduced, whereby different levels of description are recognised. It might also be helpful under some circumstances to accept inference of emotion if it is found, for example, to be a typical descriptive style of some groups. In addition, high emotion focusing was associated with ABM specificity. It would be interesting to test individuals on non-emotional cue words (in addition emotional cue words), to test whether high EF individuals still demonstrate specific recall outwith their area of focal interest. EF is a new measure so no data are available to validate its use and the division used here to differentiate high, medium and low EF was selected only as a prudent statistical division. In future, more sophisticated coding and classification categories could be explored. 
Regarding other caveats or limitations of the present study, it was important in investigating the possible mechanisms through which overgeneral recall may develop and act as a vulnerability marker for the etiology or maintenance of depression, that we test a sample vulnerable to clinical depression but not suffering from it. However, it is not clear in the literature what the precise relationship is between dysphoria and depression. We would also anticipate that with the addition of clinical data and larger sample sizes, interactions such as that between age and dysphoria may become significant and a relationship between EF and depression would perhaps emerge. Although the present results are nonetheless interesting, replication with greater sample sizes would add to the strength of the findings; for example, some subgroups in the 3x2x2 ANOVA design were small thus leading to reduced power in our investigation of the higher order EF interactions. Finally, as noted, this is the first study to use the standard ABM task in this age range. Although all children retrieved appropriate content memories to all cue words and were able to produce specific memories to at least some cue words, the lack of established validity and reliability data for this measure in children should be acknowledged as a limitation of the study.

In summary, the results of this study highlight the importance of testing children's memory with valenced emotional stimuli. As already highlighted, a key finding was that younger children tended to retrieve relatively more overgeneral memories particularly in response to negative cue words while dysphoric children demonstrated overgeneral positive recall. It may be of interest in future studies to track this valence effect through development to see what role it may play in the later onset of depression. An understanding of the nature and development of normal valenced biases in childhood clearly have important implications for understanding the development of depressogenic biases. This study has attempted to examine the development of 
valenced $A B M$ specificity as one such bias. Looking at ABM specificity from this developmental point of view should help us better understand the existence of overgeneral memory in adults and psychopathology. The results of the current study also suggested that dysphoria affects ABM in children, not by increasing availability of negative memories but by decreasing the availability of positive specific memories. It is unclear what underlying processes are responsible for such effects in the ABM literature. It is likely that more than one mechanism, bias or effect is in operation at any given time. Further work is needed to unravel the contributions and roles of associated variables. Moreover, examining differential bias across age is essential. It may be for example that such depressive biases originate with deficits in processing of positive information but spread to include negative information processing biases with age or failing intervention.

\section{References}

Beck, A.T., Rush, A.J., Shaw, B.F. \& Emery, G. (1979) Cognitive Therapy of Depression, New York: Guilford Press.

Bower, G. H. (1981). Mood and Memory. American Psychologist, 36, 129-148.

Brewin, C. H, Reynolds, M. and Tata, P. (1999) Autobiographical Processes and the Course of Depression, Journal of Abnormal Psychology, 108 (3) 511-517.

Brittlebank, A.D., Scott, J. Williams, J.M.G. and Ferrier, (1993) Autobiographical Memory in Depression: State or Trait Marker? British Journal of Psychiatry, 162, $118-121$. 
Conway, M.A. and Pleydell-Pearce, C.W. (2000) The Construction of Autobiographical Memories in the Self-Memory System, Psychological Review, 107 (2) 261-288.

Dalgleish, T., Yiend, J., Tchanturia, K., Serpell, L., Hems, S., De Silva, P. and Treasure, J. (2003) Self-reported Parental Abuse Relates to Autobiographical Memory Style in Patients with Eating Disorders, Emotion, 3, 3, 211-222.

de Decker, A., Hermans, D., Raes, F. and Eelen, P. (2003) Autobiographical Memory Specificity and Trauma in Inpatient Adolescents, Journal of Clinical Child and Adolescent Psychology, 32 (1) 22-31.

Drummond L.E., Dritschel B., Astell, A. and O’Carroll, R.E. (2005) The Influence of Age and Affect on the Perception of Facial Emotion: A study of 6-11 year olds. Manuscript submitted for publication.

Foa, E.B.and Kozak, M.J. (1986) emotional Processing of Fear- Exposure to Corrective Information, Psychological Bulletin, 99 (1) 20-35.

Gathercole, S.E. (1998) The Development of Memory, Journal of Child Psychology and Pyschiatry, 39 (1) 3-27.

Howe, M.L. and Courage, M.L. (1993) On Resolving the Enigma of Infantile Amnesia, Psychological Bulletin, 113, 305-326.

Howe, M.L., and Courage, M.L. (1997) The Emergence and Early Development of Autobiographical Memory, Psychological Review, 104 (3), 499-523

Kaslow, N. J., Tannenbaum, R. L. and Seligman, M. E. P. (1978). The KASTAN: A children's Attributional Styles Questionnaire. (Unpublished manuscript, University of Pennsylvania.)

Kovacs, M. (1992) Children’s Depression Inventory, Multi-Health Systems Inc, New York. 
Kuyken, W. and Brewin, C. R. (1995). Autobiographical Memory Functioning in Depression and Reports of Early Abuse. Journal of Abnormal Psychology, 104 (4), 585-591.

Le Gal, T., and Bruce, V. (1999). Cognitive Aspects of Emotional Expression Processing. University of Stirling, Stirling.

Mackinger, H.F., Pachinger, M.M, Leibetseder, M.M. and Fartacek, R.R. (2000) Autobiographical Memories in Women Remitted from Major Depression, Journal of Abnormal Psychology, 109 (2) 331-334.

Nelson, K. (1993) The Psychological and Social Origins of Autobiographical Memory, Psychological Science, 4, 7-14.

Neshat-Doost, H.T., Taghavi, M.R., Moradi, A.R., Yule, W. and Dalgleish, T. (1998) Memory for Emotional Trait Adjectives in Clinically Depressed Youth, Journal of Abnormal Psychology, 107 (4) 642-650.

Nolen-Hoesksema, S., Girgus, J. S. and Seligman, M.E.P. (1992) Predictors and Consequences of Childhood Depressive Symptoms- A 5-Year Longitudinal Study. Journal of Abnormal Psychology, 101 (3) 405-422.

Orbach, Y, Lamb, M.E., Sternberg, K. J., Williams, J.M.G., and Dawud-Noursi, S.( 2001) The Effects of being a Victim or Witness of Family Violence on the Retrieval of Autobiographical Memories. Chhild Abuse and Neglect, 25, 1427-1437.

Park, R.J., Goodyer, I.M. and Teasdale, J.D. (2002) Categoric overgeneral autobiographical memory in adolescents with major depressive disorder. Psychological Medicine, 32, 2, 267-276.

Power. M. and Dalgleish. T. (1997) Cognition and Emotion From Order to Disorder. Hove and New York: Psychology Press 
Segal, Z.V., Williams J.M.G., Teasdale, J.D. and Gemar, M. (1996) A Cognitive Science Perspective on Kindling and Episode Sensitization in Recurrent Affective Disorder, Psychological Medicine, 26 (2), 371-380.

Swales, M.A., Wood, P. and Williams, J.M.G. (2001) Specificity of Autobiographical Memory and Mood disturbance in Adolescents, Cognition and Emotion, 15 (3), 321-331.

Teasdale, J.D. and Barnard, P.J. (1993) Affect, Cognition and Change: Re-modelling Depressive Thought. Hove: Lawrence Erlbaum Associates.

Van Vreeswijk, M.F. and de Wilde, E.J. (2004) Autobiographical Memory Specificity, Psychopathology, Depressed Mood and the use of the Autobiographical Memory Test: A Meta-Analysis, Behaviour Research and Therapy, 42 (6) 731-743.

Williams, J.M.G. and Broadbent, K. (1986) Autobiographical Memory in Suicide Attempters, Journal of Abnormal Psychology, 95 (2) 144-149.

Williams, J.M.G., Teasdale, J.D., Segal, Z.V. and Soulsby, J. (2000) Mindfulness-based Cognitive Therapy reduces Overgeneral Autobiographical Memory in formerly Depressed Patients, Journal of Abnormal Psychology, 109 (1) 150-155.

Williams, J.M.G. (1996) Depression and the Specificity of Autobiographical Memory. In D. Rubin (Ed.), Remembering Our Past: Studies in Autobiographical Memory (pp. 244267). Cambridge, England: Cambridge University Press. 
Table 1.

Mean Specific Positive and Negative ABM scores for Dysphoric and Non-dysphoric children grouped by Age (standard deviations are presented in parentheses

\begin{tabular}{|c|c|c|c|c|c|c|c|c|c|}
\hline \multirow{2}{*}{$\begin{array}{l}\text { Cue } \\
\text { Valence }\end{array}$} & \multicolumn{3}{|c|}{$\begin{array}{l}7-8 \text { yrs } \\
(n=35)\end{array}$} & \multicolumn{3}{|c|}{$\begin{array}{c}10-11 \text { yrs } \\
(n=35)\end{array}$} & \multicolumn{3}{|c|}{$\begin{array}{c}\text { Total } \\
(n=70)\end{array}$} \\
\hline & Positive & Negative & Total & Positive & Negative & Total & Positive & Negative & Total \\
\hline $\begin{array}{l}\text { Non- } \\
\text { dysphoric } \\
(n=56)\end{array}$ & $\begin{array}{c}2.97 \\
(1.27)\end{array}$ & $\begin{array}{c}0.93 \\
(1.25)\end{array}$ & $\begin{array}{c}3.90 \\
(2.08)\end{array}$ & $\begin{array}{c}4.48 \\
(0.85)\end{array}$ & $\begin{array}{c}4.22 \\
(1.15)\end{array}$ & $\begin{array}{c}8.70 \\
(1.30)\end{array}$ & $\begin{array}{c}3.70 \\
(1.32)\end{array}$ & $\begin{array}{c}2.51 \\
(2.04)\end{array}$ & $\begin{array}{c}6.21 \\
(2.98)\end{array}$ \\
\hline $\begin{array}{l}\text { Dysphoric } \\
(n=14)\end{array}$ & $\begin{array}{c}1.67 \\
(1.21)\end{array}$ & $\begin{array}{c}2.00 \\
(2.37)\end{array}$ & $\begin{array}{c}3.67 \\
(3.14)\end{array}$ & $\begin{array}{c}2.88 \\
(0.64)\end{array}$ & $\begin{array}{c}3.00 \\
(1.51)\end{array}$ & $\begin{array}{c}5.88 \\
(1.55)\end{array}$ & $\begin{array}{c}2.36 \\
(1.10)\end{array}$ & $\begin{array}{c}2.57 \\
(1.91)\end{array}$ & $\begin{array}{c}4.93 \\
(2.53)\end{array}$ \\
\hline $\begin{array}{l}\text { Total } \\
(n=70)\end{array}$ & $\begin{array}{c}2.74 \\
(1.34)\end{array}$ & $\begin{array}{c}1.11 \\
(1.51)\end{array}$ & $\begin{array}{c}3.86 \\
(2.24)\end{array}$ & $\begin{array}{c}4.11 \\
(1.05)\end{array}$ & $\begin{array}{c}3.94 \\
(1.33)\end{array}$ & $\begin{array}{c}8.06 \\
(1.80)\end{array}$ & $\begin{array}{c}3.43 \\
(1.38)\end{array}$ & $\begin{array}{c}2.53 \\
(2.00)\end{array}$ & $\begin{array}{c}5.96 \\
(2.92)\end{array}$ \\
\hline
\end{tabular}


Table 2.

Mean Positive and Negative Specific ABMs Recalled by Children within Emotion-Focusing and Age groups (standard deviations are presented in parentheses)

\begin{tabular}{|c|c|c|c|c|c|c|c|c|c|}
\hline & & & & & EF & & & & \\
\hline & & $\begin{array}{l}\text { Low } \\
n=19)\end{array}$ & & & $\begin{array}{l}\text { ledium } \\
n=30)\end{array}$ & & & $\begin{array}{l}\text { High } \\
(n=21)\end{array}$ & \\
\hline Age/yrs & $\begin{array}{r}7-8 \\
(n=12)\end{array}$ & $\begin{array}{l}10-11 \\
(n=7)\end{array}$ & $\begin{array}{l}\text { Total } \\
(\mathrm{n}=19)\end{array}$ & $\begin{array}{r}7-8 \\
(n=16)\end{array}$ & $\begin{array}{l}10-11 \\
(n=14)\end{array}$ & $\begin{array}{l}\text { Total } \\
(\mathrm{n}=30)\end{array}$ & $\begin{array}{r}7-8 \\
(n=7)\end{array}$ & $\begin{array}{l}10-11 \\
(n=14)\end{array}$ & $\begin{array}{l}\text { Total } \\
(\mathrm{n}=21)\end{array}$ \\
\hline Negative & $\begin{array}{r}0.5 \\
(1.00)\end{array}$ & $\begin{array}{r}2.42 \\
(1.27)\end{array}$ & $\begin{array}{r}1.21 \\
(1.44)\end{array}$ & $\begin{array}{r}0.75 \\
(0.86)\end{array}$ & $\begin{array}{r}4.00 \\
(1.18)\end{array}$ & $\begin{array}{r}2.27 \\
(1.93)\end{array}$ & $\begin{array}{r}3.00 \\
(2.00)\end{array}$ & $\begin{array}{r}4.64 \\
(0.84)\end{array}$ & $\begin{array}{r}4.10 \\
(1.51)\end{array}$ \\
\hline Positive & $\begin{array}{r}2.25 \\
(1.42)\end{array}$ & $\begin{array}{r}4.14 \\
(1.06)\end{array}$ & $\begin{array}{r}2.95 \\
(1.58)\end{array}$ & $\begin{array}{r}2.87 \\
(1.15)\end{array}$ & $\begin{array}{r}4.36 \\
(0.74)\end{array}$ & $\begin{array}{r}3.57 \\
(1.22)\end{array}$ & $\begin{array}{r}3.29 \\
(1.50)\end{array}$ & $\begin{array}{r}3.86 \\
(1.29)\end{array}$ & $\begin{array}{r}3.67 \\
(1.35)\end{array}$ \\
\hline
\end{tabular}

